Stud. Univ. Babeş-Bolyai Math. 66(2021), No. 1, 197-209

DOI: 10.24193/subbmath.2021.1.16

\title{
Permanent solutions for some motions of UCM fluids with power-law dependence of viscosity on the pressure
}

\author{
Constantin Fetecau and Abdul Rauf
}

Dedicated to Professor Gheorghe Moroşanu on the occasion of his 70th anniversary.

\begin{abstract}
Steady motion of two types of incompressible Maxwell fluids with power-law dependence of viscosity on the pressure is analytically studied between infinite horizontal parallel plates when the gravity effects are taken into consideration. Simple and exact expressions are established for the permanent components of starting solutions corresponding to two oscillatory motions induced by the lower plate that oscillates in its plane. Such solutions are very important for the experimentalists who want to eliminate the transients from their experiments. The similar solutions for the simple Couette flow of the same fluids, as well as the permanent solutions corresponding to ordinary incompressible Maxwell fluids performing the same motions, are obtained as limiting cases of general solutions. The convergence of starting solutions to their permanent components as well as the influence of physical parameters on the fluid motion is graphically underlined and discussed.
\end{abstract}

Mathematics Subject Classification (2010): 76A05.

Keywords: Permanent solutions, UCM fluids, Pressure-dependent viscosity.

\section{Introduction}

Generally, in isothermal processes, the fluid viscosity depends on the flow conditions and particularly on the shear-rate and pressure. The first who remarked that the fluid viscosity could depend on the pressure was Stokes in his seminal work [21]. He also delineated motions in which the viscosity can be considered constant. Later, experiments by Barus [2], Bridgman [3], Griest et al. [9], Bair et al. [1], Prusa et al. [16] and so on certified this dependence. A linear dependence of viscosity on the pressure was proposed by Barus [2] for low to medium pressure difference. At large pressure differences, power or exponential law seems to be more suitable. In any case, exact 
solutions for steady (permanent) or unsteady motions of non-Newtonian fluids with the pressure-dependent viscosity are lack in the existing literature excepting those of Housiadas $[10,11]$ corresponding to a linear law of viscosity on the pressure.

In the following, we shall determine simple closed form solutions for the permanent velocity fields corresponding to some unsteady motions of Maxwell fluids with power-law dependence of the viscosity on the pressure. It is worth to point out the fact that high pressure differences appear in many engineering applications such as polymer processing operations [5], fluid film lubrication [22], microfluidics [4], pharmaceutical tablet manufacturing, food processing and geophysics [20]. Techniques for measuring the pressure dependent viscosity and of the pressure-viscosity coefficient can be found in the work of Goubert et al. [8], respectively Park et al. [14]. An extensive literature regarding experimental and theoretical studies on these fluids was provided by Malek and Rajagopal [13].

On the other hand, the gravity effects are important in many flows of the fluids with practical applications. They are stronger in the case of fluid motions in which the gravity acts along the direction in which the pressure varies. The first exact solutions for steady motions of the Newtonian fluids with pressure-dependent viscosity are those of Rajagopal $[17,18]$ between parallel plates or over an inclined plane due to the gravity. Exact expressions in terms of the Kelvin functions have been established by Prusa [15] for the solutions of modified Stokes problems of the same fluids. General solutions for the same motions of the incompressible Newtonian fluids with powerlaw or exponential dependence of viscosity on the pressure have been determined by Rajagopal et al. [19] and Fetecau and Vieru [7].

In the present work we provide the first exact and simple expressions for permanent solutions corresponding to two oscillatory motions of incompressible Maxwell fluids with exponential dependence of viscosity on the pressure between infinite horizontal parallel plates. The fluid motion is generated by the lower plate that oscillates in its plane. The similar solutions for the simple Couette flow of the same fluids as well as some known solutions for the Newtonian fluids performing the same motions are obtained as limiting cases of general solutions. Such solutions are important for the experimentalists who want to eliminate the transients from their experiments. In addition, they can be also used as tests to verify different numerical methods that are used to study complex flow problems. Finally, the convergence of starting solutions (numerical solutions) to their permanent components, as well as the effect of physical parameters on the fluid motion, is graphically underlined and discussed.

\section{Statement of the problem}

Let us consider an incompressible upper-convected Maxwell (UCM) fluid with power-law dependence of viscosity on the pressure at rest between two infinite horizontal parallel plates at the distance $d$ apart. Its constitutive equations, as it results 
from [12], are

$$
\begin{aligned}
& \mathbf{T}=-p \mathbf{I}+\mathbf{S}, \quad \mathbf{S}+\lambda \frac{\delta \mathbf{S}}{\delta t}=\eta(p) \mathbf{A} \\
& \text { with } \operatorname{tr} \mathbf{A}=0 \text { and } \frac{\delta \mathbf{S}}{\delta t}=\frac{d \mathbf{S}}{d t}-\mathbf{L S}-\mathbf{S L}^{\mathrm{T}},
\end{aligned}
$$

where $\mathbf{T}$ is the Cauchy stress tensor, $\mathbf{S}$ the extra-stress tensor, $\mathbf{I}$ is the unit tensor, $\mathbf{A}$ is the first Rivlin-Ericksen tensor, $\mathbf{L}$ is the gradient of the velocity vector $\mathbf{u}, p$ is the Lagrange multiplier, $\lambda$ is the relaxation time and $\eta(p)$ is the fluid viscosity which depends on the pressure.

In the next, we determine exact expressions for the permanent (steady-state or long-time) solutions corresponding to some motions of the incompressible UCM fluids with power-law dependence of viscosity on the pressure of the forms

$$
\eta(p)=\mu\left[\alpha\left(p-p_{0}\right)+1\right]^{2} \quad \text { or } \quad \eta(p)=\mu\left[\alpha\left(p-p_{0}\right)+1\right]^{1 / 2},
$$

where $\mu$ is the fluid viscosity at the reference pressure $p_{0}$ and the positive constant $\alpha$ is the dimensional pressure-viscosity coefficient. The gravitational effects will be also taken into consideration. If $\alpha=0, \eta(p)=\mu$ and Eqs. (2.1) correspond to the ordinary UCM fluids. If both $\alpha$ and $\lambda$ are zero, the governing equations (2.1) correspond to incompressible ordinary Newtonian fluids.

At the moment $t=0^{+}$the lower plate begins to oscillate in its plane according to

$$
\mathbf{u}=U \cos (\omega t) \mathbf{i} \quad \text { or } \quad \mathbf{u}=U \sin (\omega t) \mathbf{i},
$$

where $\mathbf{i}$ is the unit vector along the $x$-direction of a suitable Cartesian coordinate system $x, y$ and $z$ while $U$ and $\omega$ are the amplitude, respectively the frequency of the oscillations. Due to the shear the fluid is gradually moved and we are looking for a solution of the form $[15,19,12]$

$$
\mathbf{u}=u(y, t) \mathbf{i}, \quad p=p(y) .
$$

Using Eqs. (2.4) in (2.1) and introducing the obtained results in the balance of linear momentum, we get the following relevant partial or ordinary differential equations $[12]$

$$
\begin{aligned}
& \tau(y, t)+\lambda \frac{\partial \tau(y, t)}{\partial t}=\eta(p) \frac{\partial u(y, t)}{\partial y}, \quad \rho \frac{\partial u(y, t)}{\partial t}=\frac{\partial \tau(y, t)}{\partial y}, \\
& \frac{d p(y)}{d y}+\rho g=0 ; 0<y<d, \quad t>0,
\end{aligned}
$$

where $\tau(y, t)$ is the non-trivial shear stress, $\rho$ is the fluid density and $g$ is the gravitational acceleration. The incompressibility condition is automatically satisfied and Eq. $(2.5)_{3}$ implies

$$
p(y)=\rho g(d-y)+p_{0} \quad \text { with } \quad p_{0}=p(d) .
$$

Eliminating $\tau(y, t)$ between Eqs. $(2.5)_{1}$ and $(2.5)_{2}$ and bearing in mind the expressions of $\eta(p)$ and $p(y)$ from Eqs. (2.2) and (2.6), we find the next governing equations

$$
\begin{aligned}
& \mu[\alpha \rho g(d-y)+1]^{2} \frac{\partial^{2} u(y, t)}{\partial y^{2}}-2 \mu \alpha \rho g[\alpha \rho g(d-y)+1] \frac{\partial u(y, t)}{\partial y} \\
& =\rho\left[\lambda \frac{\partial^{2} u(y, t)}{\partial t^{2}}+\frac{\partial u(y, t)}{\partial t}\right] ; \quad 0<y<d, t>0,
\end{aligned}
$$




$$
\begin{aligned}
& \mu \sqrt{\alpha \rho g(d-y)+1} \frac{\partial^{2} u(y, t)}{\partial y^{2}}-\frac{\mu \alpha \rho g}{2 \sqrt{\alpha \rho g(d-y)+1}} \frac{\partial u(y, t)}{\partial y} \\
& =\rho\left[\lambda \frac{\partial^{2} u(y, t)}{\partial t^{2}}+\frac{\partial u(y, t)}{\partial t}\right] ; \quad 0<y<d, \quad t>0,
\end{aligned}
$$

for the velocity field $u(y, t)$ corresponding to the motion of the two types of UCM fluids with power-law dependence of viscosity on the pressure between infinite horizontal parallel plates.

The appropriate initial and boundary conditions are given by the relations

$$
\begin{gathered}
u(y, 0)=0,\left.\quad \frac{\partial u(y, t)}{\partial t}\right|_{t=0}=0 \text { if } 0 \leq y \leq d, \\
u(0, t)=U \cos (\omega t) \text { or } u(0, t)=U \sin (\omega t), u(d, t)=0 \text { for } t>0 .
\end{gathered}
$$

Introducing the following non-dimensional variables, functions and parameters

$$
y^{*}=\frac{y}{d}, t^{*}=\frac{\nu t}{d^{2}}, \quad u^{*}=\frac{u}{U}, \quad \tau^{*}=\frac{\tau d}{\mu U}, \quad \omega^{*}=\frac{d^{2}}{\nu} \omega, \quad \alpha^{*}=\alpha \rho g d,
$$

where $\nu=\mu / \rho$ is the kinematic viscosity of the fluid and dropping out the star notation, we obtain the following two dimensionless initial and boundary value problems

$$
\begin{aligned}
& \quad[\alpha(1-y)+1]^{2} \frac{\partial^{2} u(y, t)}{\partial y^{2}}-2 \alpha[\alpha(1-y)+1] \frac{\partial u(y, t)}{\partial y} \\
& =\mathrm{We} \frac{\partial^{2} u(y, t)}{\partial t^{2}}+\frac{\partial u(y, t)}{\partial t} ; \quad 0<y<1, t>0, \\
& u(y, 0)=\left.\frac{\partial u(y, t)}{\partial t}\right|_{t=0}=0,0 \leq y \leq 1 ; \\
& u(0, t)=\cos (\omega t) \text { or } \sin (\omega t), u(1, t)=0 \text { for } t>0,
\end{aligned}
$$

and

$$
\begin{gathered}
\sqrt{\alpha(1-y)+1} \frac{\partial^{2} u(y, t)}{\partial y^{2}}-\frac{\alpha}{2 \sqrt{\alpha(1-y)+1}} \frac{\partial u(y, t)}{\partial y} \\
=\mathrm{We} \frac{\partial^{2} u(y, t)}{\partial t^{2}}+\frac{\partial u(y, t)}{\partial t} ; \quad 0<y<1, \quad t>0, \\
u(y, 0)=\left.\frac{\partial u(y, t)}{\partial t}\right|_{t=0}=0,0 \leq y \leq 1 ; \\
u(0, t)=\cos (\omega t) \text { or } \sin (\omega t), u(1, t)=0 \text { for } t>0,
\end{gathered}
$$

where We $=\lambda \nu / d^{2}=\lambda /\left(d^{2} / \nu\right)$ is the Weissenberg number (the ratio of the relaxation time of the fluid and a characteristic time scale).

\section{Solution}

In the following, in order to avoid confusion, we shall denote by $u_{c}(y, t)$ and $u_{s}(y, t)$ the starting solutions of the dimensionless initial and boundary value problems characterized by Eqs. (2.12), (2.13) or (2.14), (2.15). Generally, these solutions can be written as sums of their permanent and transient components, namely

$$
u_{c}(y, t)=u_{c p}(y, t)+u_{c t}(y, t), \quad u_{s}(y, t)=u_{s p}(y, t)+u_{s t}(y, t) .
$$


Some time after the motion initiation, the fluid moves according to starting solutions. After this time when the transients disappear or can be neglected, its movement is characterized by the permanent solutions $u_{c p}(y, t)$ or $u_{s p}(y, t)$. In practice, the experimentalists want to know the required time to reach the permanent state. This is the time after which the fluid flows according to the permanent solutions. In order to determine it, at least the permanent solutions corresponding to a given motion have to be known. This is the reason that, in this section, we shall determine closed form expressions for these solutions only.

3.1. Case $\eta(p)=\mu[\alpha \rho g(d-y)+1]^{2}$

To determine both permanent solutions in the same time, we use the complex velocity

$$
u_{p}(y, t)=u_{c p}(y, t)+i u_{s p}(y, t),
$$

where $i$ is the imaginary unit. This velocity has to satisfy the partial differential equation

$$
\begin{aligned}
& {[\alpha(1-y)+1]^{2} \frac{\partial^{2} u_{p}(y, t)}{\partial y^{2}}-2 \alpha[\alpha(1-y)+1] \frac{\partial u_{p}(y, t)}{\partial y}} \\
& =\mathrm{We} \frac{\partial^{2} u_{p}(y, t)}{\partial t^{2}}+\frac{\partial u_{p}(y, t)}{\partial t} ; \quad 0<y<1, \quad t \in R,
\end{aligned}
$$

with the boundary conditions

$$
u_{p}(0, t)=\mathrm{e}^{i \omega t}, \quad u_{p}(1, t)=0 ; \quad t \in R .
$$

Making the following suitable change of the spatial variable

$$
y=\frac{\alpha+1-\mathrm{e}^{r}}{\alpha} \text { or equivalently } r=\ln [\alpha(1-y)+1],
$$

Eq. (3.3) takes the simpler form

$$
\begin{aligned}
& \alpha^{2}\left[\frac{\partial^{2} u_{p}(r, t)}{\partial r^{2}}+\frac{\partial u_{p}(r, t)}{\partial r}\right]=\mathrm{We} \frac{\partial^{2} u_{p}(r, t)}{\partial t^{2}}+\frac{\partial u_{p}(r, t)}{\partial t} ; \\
& 0<r<a, \quad t>0,
\end{aligned}
$$

where $a=\ln (\alpha+1)$. The corresponding boundary conditions are

$$
u_{p}(0, t)=0, \quad u_{p}(a, t)=\mathrm{e}^{i \omega t} ; \quad t \in R .
$$

Following [12], we are looking for a solution of the form

$$
u_{p}(r, t)=U(r) \mathrm{e}^{i \omega t} ; \quad r \in(0, a), \quad t \in R .
$$

Substituting $u_{p}(r, t)$ from Eq. (3.8) in (3.6), we find that the unknown function $U(r)$ has to satisfy the following boundary value problem

$$
\alpha^{2}\left[U^{\prime \prime}(r)+U^{\prime}(r)\right]+\omega(\omega \mathrm{We}-i) U(r)=0 ; \quad U(0)=0, \quad U(a)=1 .
$$

The solution of this boundary value problem is given by the next equality

$$
U(r)=\frac{\mathrm{e}^{r_{2} r}-\mathrm{e}^{r_{1} r}}{\mathrm{e}^{r_{2} a}-\mathrm{e}^{r_{1} a}} ; \quad r_{1,2}=\frac{-1 \pm \sqrt{1-4 \omega(\omega \mathrm{We}-i) / \alpha^{2}}}{2} .
$$


Consequently, the complex velocity field $u_{p}(y, t)$ is given by

$$
u_{p}(y, t)=\frac{[\alpha(1-y)+1]^{r_{2}}-[\alpha(1-y)+1]^{r_{1}}}{(\alpha+1)^{r_{2}}-(\alpha+1)^{r_{1}}} e^{i \omega t} ; 0<y<1, t>0,
$$

and the dimensionless permanent velocities $u_{c p}(y, t)$ and $u_{s p}(y, t)$ have the expressions

$$
\begin{aligned}
& u_{c p}(y, t)=\operatorname{Re}\left\{\frac{[\alpha(1-y)+1]^{r_{2}}-[\alpha(1-y)+1]^{r_{1}}}{(\alpha+1)^{r_{2}}-(\alpha+1)^{r_{1}}} \mathrm{e}^{i \omega t}\right\}, \\
& u_{s p}(y, t)=\operatorname{Im}\left\{\frac{[\alpha(1-y)+1]^{r_{2}}-[\alpha(1-y)+1]^{r_{1}}}{(\alpha+1)^{r_{2}}-(\alpha+1)^{r_{1}}} \mathrm{e}^{i \omega t}\right\},
\end{aligned}
$$

where $\operatorname{Re}$ and $\mathrm{Im}$ denote the real, respectively the imaginary part of that which follows.

3.2. Case $\eta(p)=\mu\left[\alpha\left(p-p_{0}\right)+1\right]^{1 / 2}$

In this case the corresponding dimensionless complex velocity $u_{p}(y, t)$ has to satisfy the following partial differential equation

$$
\begin{aligned}
& \sqrt{\alpha(1-y)+1} \frac{\partial^{2} u_{p}(y, t)}{\partial y^{2}}-\frac{\alpha}{2 \sqrt{\alpha(1-y)+1}} \frac{\partial u_{p}(y, t)}{\partial y} \\
& =\mathrm{We} \frac{\partial^{2} u_{p}(y, t)}{\partial t^{2}}+\frac{\partial u_{p}(y, t)}{\partial t} ; \quad 0<y<1, \quad t \in R,
\end{aligned}
$$

with the same boundary conditions (3.4). Making the change of spatial variable

$$
y=\frac{\alpha+1-r^{2}}{\alpha} \text { or equivalently } r=\sqrt{\alpha(1-y)+1},
$$

we attain to the next boundary value problem

$$
\frac{\alpha^{2}}{4 r} \frac{\partial^{2} u_{p}(r, t)}{\partial r^{2}}=\mathrm{We} \frac{\partial^{2} u_{p}(r, t)}{\partial t^{2}}+\frac{\partial u_{p}(r, t)}{\partial t} ; \quad 1<r<b, t>0,
$$

where the constant $b=\sqrt{\alpha+1}$.

Looking again for a solution of the form (3.8), we find that the corresponding function $U(r)$ has to satisfy the following boundary value problem

$$
\alpha^{2} U^{\prime \prime}(r)+4 \gamma r U(r)=0 ; \quad U(1)=0, \quad U(b)=1,
$$

where $\gamma=\omega(\omega \mathrm{We}-i)$. The equation (3.17) is an ordinary differential equation of Airy type whose general solution is of the form

$$
U(r)=\sqrt{r}\left[C_{1} J_{1 / 3}\left(\frac{4 r}{3 \alpha} \sqrt{\gamma r}\right)+C_{2} Y_{1 / 3}\left(\frac{4 r}{3 \alpha} \sqrt{\gamma r}\right)\right],
$$

where $C_{1}$ and $C_{2}$ are arbitrary constants. On the basis of the boundary conditions $(3.17)_{2}$ and $(3.17)_{3}$, it immediately results that

$$
U(r)=\frac{\sqrt{r}}{\sqrt{b}} \frac{Y_{\frac{1}{3}}\left(\frac{4 \sqrt{\gamma}}{3 \alpha}\right) J_{\frac{1}{3}}\left(\frac{4 r}{3 \alpha} \sqrt{\gamma r}\right)-J_{\frac{1}{3}}\left(\frac{4 \sqrt{\gamma}}{3 \alpha}\right) Y_{\frac{1}{3}}\left(\frac{4 r}{3 \alpha} \sqrt{\gamma r}\right)}{Y_{\frac{1}{3}}\left(\frac{4 \sqrt{\gamma}}{3 \alpha}\right) J_{\frac{1}{3}}\left(\frac{4 b}{3 \alpha} \sqrt{\gamma b}\right)-J_{\frac{1}{3}}\left(\frac{4 \sqrt{\gamma}}{3 \alpha}\right) Y_{\frac{1}{3}}\left(\frac{4 b}{3 \alpha} \sqrt{\gamma b}\right)} .
$$


Consequently, the dimensionless velocity fields corresponding to this problem are

$$
\begin{aligned}
& u_{c p}(y, t)=\frac{\sqrt{r}}{\sqrt{b}} \operatorname{Re}\left\{\frac{Y_{\frac{1}{3}}\left(\frac{4 \sqrt{\gamma}}{3 \alpha}\right) J_{\frac{1}{3}}\left(\frac{4 r}{3 \alpha} \sqrt{\gamma r}\right)-J_{\frac{1}{3}}\left(\frac{4 \sqrt{\gamma}}{3 \alpha}\right) Y_{\frac{1}{3}}\left(\frac{4 r}{3 \alpha} \sqrt{\gamma r}\right)}{Y_{\frac{1}{3}}\left(\frac{4 \sqrt{\gamma}}{3 \alpha}\right) J_{\frac{1}{3}}\left(\frac{4 b}{3 \alpha} \sqrt{\gamma b}\right)-J_{\frac{1}{3}}\left(\frac{4 \sqrt{\gamma}}{3 \alpha}\right) Y_{\frac{1}{3}}\left(\frac{4 b}{3 \alpha} \sqrt{\gamma b}\right)} \mathrm{e}^{i \omega t}\right\}, \\
& u_{s p}(y, t)=\frac{\sqrt{r}}{\sqrt{b}} \operatorname{Im}\left\{\frac{Y_{\frac{1}{3}}\left(\frac{4 \sqrt{\gamma}}{3 \alpha}\right) J_{\frac{1}{3}}\left(\frac{4 r}{3 \alpha} \sqrt{\gamma r}\right)-J_{\frac{1}{3}}\left(\frac{4 \sqrt{\gamma}}{3 \alpha}\right) Y_{\frac{1}{3}}\left(\frac{4 r}{3 \alpha} \sqrt{\gamma r}\right)}{Y_{\frac{1}{3}}\left(\frac{4 \sqrt{\gamma}}{3 \alpha}\right) J_{\frac{1}{3}}\left(\frac{4 b}{3 \alpha} \sqrt{\gamma b}\right)-J_{\frac{1}{3}}\left(\frac{4 \sqrt{\gamma}}{3 \alpha}\right) Y_{\frac{1}{3}}\left(\frac{4 b}{3 \alpha} \sqrt{\gamma b}\right)} \mathrm{e}^{i \omega t}\right\},
\end{aligned}
$$

where $r=\sqrt{\alpha(1-y)+1}$. These solutions, as well as those given by Eqs. (3.12) and (3.13), are independent of initial conditions $(2.15)_{1}$ and $(2.15)_{2}$ but satisfy the boundary conditions and the corresponding governing equations (2.12), respectively $(2.14)$.

\section{Limiting cases}

In order to obtain the steady solutions corresponding to the simple Couette flow of the same fluids, as well as the permanent solutions for incompressible ordinary UCM fluids performing the same motions or to recover some known results from the existing literature, we shall consider in this section three special cases.

\subsection{Case $\omega \rightarrow 0$ (Simple Couette flow)}

Taking the limit of the permanent solution $u_{c p}(y, t)$ given by Eq. (3.12) when $\omega \rightarrow 0$ and using the asymptotic approximations

$$
J_{\nu}(z) \approx \frac{z^{\nu}}{2^{\nu} \Gamma(\nu+1)}, \quad Y_{\nu}(z) \approx-\frac{2^{\nu} \Gamma(\nu)}{\pi z^{\nu}} \text { for } \nu>0 \text { and } z<<1,
$$

for $u_{c p}(y, t)$ given by Eq. (3.20), we recover the steady solutions [[6], Eqs. (44)]

$$
u_{C p}(y)=\frac{(\alpha+1)(1-y)}{\alpha(1-y)+1}, \quad \text { respectively } u_{C p}(y)=\frac{\sqrt{\alpha(1-y)+1}-1}{\sqrt{\alpha+1}-1},
$$

corresponding to the simple Couette flow of the two types of incompressible Newtonian fluids with power-law dependence of viscosity on the pressure. This is not a surprise since the governing equations corresponding to steady motions of incompressible Newtonian or UCM fluids with/without pressure dependent viscosity are identical. It is important to point out the fact that the dimensionless steady solutions given by Eqs. (4.2) can be directly obtained from the corresponding boundary value problems. They correspond to the fluid motion induced by the lower plate that is moving in its plane with the constant velocity $U$.

\subsection{Case $\alpha \rightarrow 0$ (Flows of ordinary incompressible UCM fluids)}

The governing equation corresponding to unsteady motions of incompressible ordinary UCM fluids between infinite horizontal parallel plates, namely

$$
\mathrm{We} \frac{\partial^{2} u(y, t)}{\partial t^{2}}+\frac{\partial u(y, t)}{\partial t}=\frac{\partial^{2} u(y, t)}{\partial y^{2}} ; \quad 0<y<1, t>0
$$


is obtained making $\alpha \rightarrow 0$ in any one of the equations (2.12) or (2.14). Consequently, the velocity fields $u_{O c p}(y, t)$ and $u_{O s p}(y, t)$ corresponding to unsteady motions of these fluids generated by cosine, respectively sine oscillations of the lower plate can be obtained as limiting cases of the solutions $u_{c p}(y, t)$ and $u_{s p}(y, t)$ given by Eqs. (3.12), (3.13) or (3.20), (3.21). Using the well known asymptotic approximations

$$
\begin{aligned}
& J_{\nu}(z) \approx \sqrt{\frac{2}{\pi z}} \cos \left[z-\frac{(2 \nu+1) \pi}{4}\right], \\
& Y_{\nu}(z) \approx \sqrt{\frac{2}{\pi z}} \sin \left[z-\frac{(2 \nu+1) \pi}{4}\right] \text { for } z>>1,
\end{aligned}
$$

in Eqs. (3.20) and (3.21), it is easy to show that for small enough values of the dimensionless pressure-viscosity coefficient $\alpha$

$$
\begin{aligned}
& u_{c p}(y, t) \approx \frac{\sqrt[8]{\alpha+1}}{\sqrt[8]{\alpha(y-1)+1}} \operatorname{Re}\left\{\frac{\sin \left\{\frac{4 \sqrt{\gamma}}{3 \alpha}\left[1-\sqrt[4]{[\alpha(1-y)+1]^{3}}\right]\right\}}{\sin \left\{\frac{4 \sqrt{\gamma}}{3 \alpha}\left[1-\sqrt[4]{(\alpha+1)^{3}}\right]\right\}} \mathrm{e}^{i \omega t}\right\}, \\
& u_{s p}(y, t) \approx \frac{\sqrt[8]{\alpha+1}}{\sqrt[8]{\alpha(y-1)+1}} \operatorname{Im}\left\{\frac{\sin \left\{\frac{4 \sqrt{\gamma}}{3 \alpha}\left[1-\sqrt[4]{[\alpha(1-y)+1]^{3}}\right]\right\}}{\sin \left\{\frac{4 \sqrt{\gamma}}{3 \alpha}\left[1-\sqrt[4]{(\alpha+1)^{3}}\right]\right\}} \mathrm{e}^{i \omega t}\right\} .
\end{aligned}
$$

Now, using the Maclaurin series expansions for $[1+\alpha(1-y)]^{3 / 4}$ and $(1+\alpha)^{3 / 4}$ in Eqs. (4.5) and (4.6) and taking their limits when $\alpha \rightarrow 0$, we get the permanent solutions

$$
\begin{aligned}
& u_{O c p}(y, t)=\operatorname{Re}\left\{\frac{\sin [(1-y) \sqrt{\gamma}]}{\sin (\sqrt{\gamma})} \mathrm{e}^{i \omega t}\right\}, \\
& u_{O s p}(y, t)=\operatorname{Im}\left\{\frac{\sin [(1-y) \sqrt{\gamma}]}{\sin (\sqrt{\gamma})} \mathrm{e}^{i \omega t}\right\},
\end{aligned}
$$

corresponding to the incompressible ordinary UCM fluids performing the same motions. To the best of our knowledge, the solutions given by Eqs. (4.7) are also new in the literature. As expected, making $\alpha \rightarrow 0$ in any one of the equalities (4.2) or $\omega \rightarrow 0$ in Eq. $(4.7)_{1}$, the steady velocity field $u_{N C p}(y)=1-y$ corresponding to the simple Couette flow of incompressible ordinary Newtonian fluids is recovered.

\subsection{Case $\mathrm{We} \rightarrow 0$ (Flows of Newtonian fluids with pressure-dependent viscosity)}

By now letting We $\rightarrow 0$ in all results that have been obtained in the third section, we recover the dimensionless permanent solutions corresponding to the two types of incompressible Newtonian fluids with power-law dependence of viscosity on the pressure performing the same motions. More precisely, the permanent velocity fields $u_{c p}(y, t)$ and $u_{s p}(y, t)$ given by Eqs. (3.12), (3.13) and (3.20), (3.21) take the simplified forms $[6]$

$$
u_{N c p}(y, t)=\operatorname{Re}\left\{\frac{[\alpha(1-y)+1]^{r_{4}}-[\alpha(1-y)+1]^{r_{3}}}{(\alpha+1)^{r_{4}}-(\alpha+1)^{r_{3}}} \mathrm{e}^{i \omega t}\right\},
$$




$$
u_{N s p}(y, t)=\operatorname{Im}\left\{\frac{[\alpha(1-y)+1]^{r_{4}}-[\alpha(1-y)+1]^{r_{3}}}{(\alpha+1)^{r_{4}}-(\alpha+1)^{r_{3}}} \mathrm{e}^{i \omega t}\right\},
$$

respectively

$$
\begin{aligned}
& u_{N c p}(y, t)= \\
& \frac{\sqrt{r}}{\sqrt{b}} \operatorname{Re}\left\{\frac{Y_{\frac{1}{3}}\left(\frac{4 \sqrt{-i \omega}}{3 \alpha}\right) J_{\frac{1}{3}}\left(\frac{4 r}{3 \alpha} \sqrt{-i \omega r}\right)-J_{\frac{1}{3}}\left(\frac{4 \sqrt{-i \omega}}{3 \alpha}\right) Y_{\frac{1}{3}}\left(\frac{4 r}{3 \alpha} \sqrt{-i \omega r}\right)}{Y_{\frac{1}{3}}\left(\frac{4 \sqrt{-i \omega}}{3 \alpha}\right) J_{\frac{1}{3}}\left(\frac{4 b}{3 \alpha} \sqrt{-i \omega b}\right)-J_{\frac{1}{3}}\left(\frac{4 \sqrt{-i \omega}}{3 \alpha}\right) Y_{\frac{1}{3}}\left(\frac{4 b}{3 \alpha} \sqrt{-i \omega b}\right)} \mathrm{e}^{i \omega t}\right\}, \\
& u_{N s p}(y, t)= \\
& \frac{\sqrt{r}}{\sqrt{b}} \operatorname{Im}\left\{\frac{Y_{\frac{1}{3}}\left(\frac{4 \sqrt{-i \omega}}{3 \alpha}\right) J_{\frac{1}{3}}\left(\frac{4 r}{3 \alpha} \sqrt{-i \omega r}\right)-J_{\frac{1}{3}}\left(\frac{4 \sqrt{-i \omega}}{3 \alpha}\right) Y_{\frac{1}{3}}\left(\frac{4 r}{3 \alpha} \sqrt{-i \omega r}\right)}{Y_{\frac{1}{3}}\left(\frac{4 \sqrt{-i \omega}}{3 \alpha}\right) J_{\frac{1}{3}}\left(\frac{4 b}{3 \alpha} \sqrt{-i \omega b}\right)-J_{\frac{1}{3}}\left(\frac{4 \sqrt{-i \omega}}{3 \alpha}\right) Y_{\frac{1}{3}}\left(\frac{4 b}{3 \alpha} \sqrt{-i \omega b}\right)} \mathrm{e}^{i \omega t}\right\},
\end{aligned}
$$

where $r_{3,4}=\frac{-1 \pm \sqrt{1+4 i \omega / \alpha^{2}}}{2}$.

\section{Numerical results, discussion and conclusions}

Generally, starting solutions corresponding to oscillatory motions of fluids can be presented as sums of their permanent and transient components. As it is known, the transient solutions tend to zero for increasing values of the time $t$. Consequently, the transients disappear in time and in practice it is important to know the time after which the fluid flows according to the permanent solution. This is the required time to reach the permanent state. To determine it, for a given motion, at least the permanent solution has to be known. This is the reason that we established here closed form expressions for the permanent solutions corresponding to some oscillatory motions of two types of incompressible UCM fluids with power-law dependence of viscosity on the pressure.

These expressions have been also used to determine the similar solutions $(4.2)_{1}$ and $(4.2)_{2}$ for the simple Couette flow of the same fluids and to provide the corresponding solutions from Eqs. (4.8)-(4.11) for ordinary incompressible UCM fluids performing the same motions. In addition, as a proof of their correctness, known solutions for the incompressible Newtonian fluids with power-law dependence of the viscosity on the pressure performing the same motions have been obtained as limiting cases of present solutions.

In order to get some physical insight of results that have been here obtained, as well as to certify their correctness, Figs 1-4 have been prepared for different values of the physical parameters and the time $t$. Figs. 1 and 2 clearly show that, as expected, the starting solutions $u_{c}(y, t)$ corresponding to the motion of the two types of incompressible UCM fluids with power-law dependence of viscosity on the pressure induced by cosine oscillations of the lower plate converge to their permanent components $u_{c p}(y, t)$ given by Eqs. (3.12) and (3.20). Furthermore, from these figures it also results that the required time to reach the permanent state diminishes for decreasing values of the Weissenberg number We. Consequently, the permanent state is rather 
obtained for oscillatory motions of Newtonian fluids as compared to UCM fluids with power-law dependence of viscosity on the pressure.

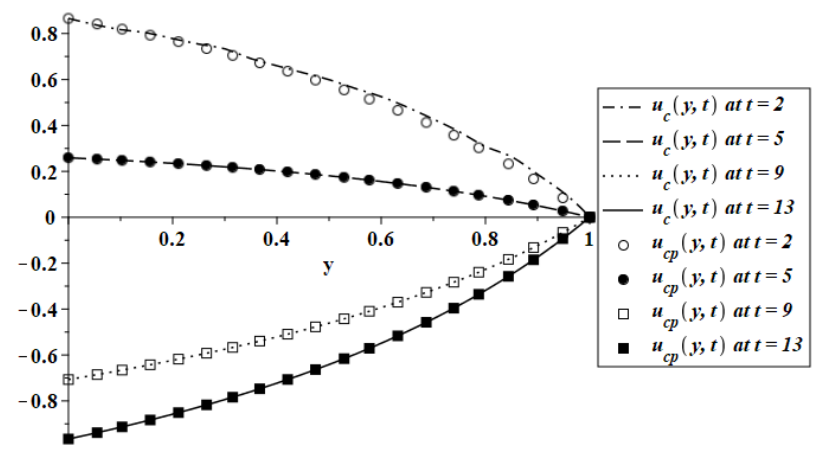

(A) $\mathrm{We}=0.3$

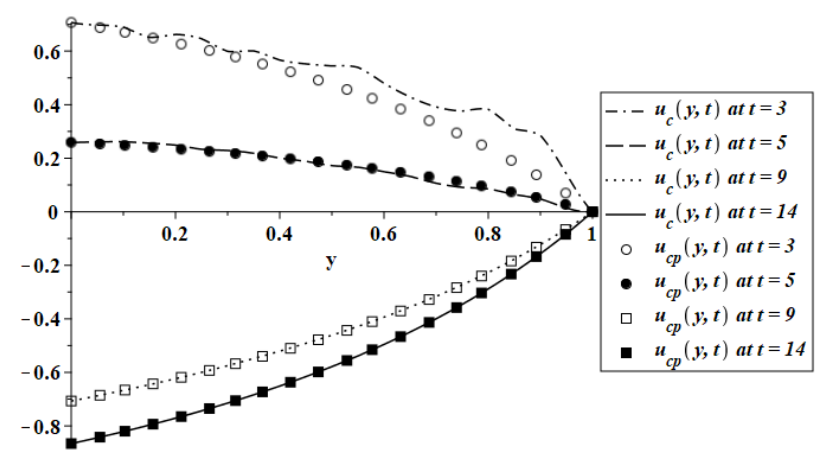

(B) $\mathrm{We}=0.7$

Figure (1) Convergence of starting solution $u_{c}(y, t)$ (numerical solution) to its permanent component $u_{c p}(y, t)$ given by Eq. (3.12) for $\alpha=0.95, \omega=\pi / 12$ and two values of We.

In the subsection 4.2 we analytically proved that, if the dimensionless pressureviscosity coefficient $\alpha \rightarrow 0$, the permanent solutions $u_{c p}(y, t)$ and $u_{s p}(y, t)$ given by Eqs. (3.20) and (3.21) tend to the permanent solutions $u_{O c p}(y, t)$, respectively $u_{O s p}(y, t)$ corresponding to the ordinary incompressible UCM fluids performing the same motions. For completion, as well as for the results validation, Figs. 3 have been prepared to show the convergence of the other permanents solutions $u_{c p}(y, t)$ and $u_{s p}(y, t)$ given by Eqs. (3.12) and (3.13) to $u_{O c p}(y, t)$ and $u_{O s p}(y, t)$. In all cases, the fluid velocity smoothly decreases from maximum values on the lower plate to the zero value on the stationary wall.

The time variations of the dimensionless mid plane velocity fields $u_{c p}(0.5, t)$ and $u_{s p}(0.5, t)$ given by Eqs. (3.12) and (3.13) have been depicted in Figs. 4 for 


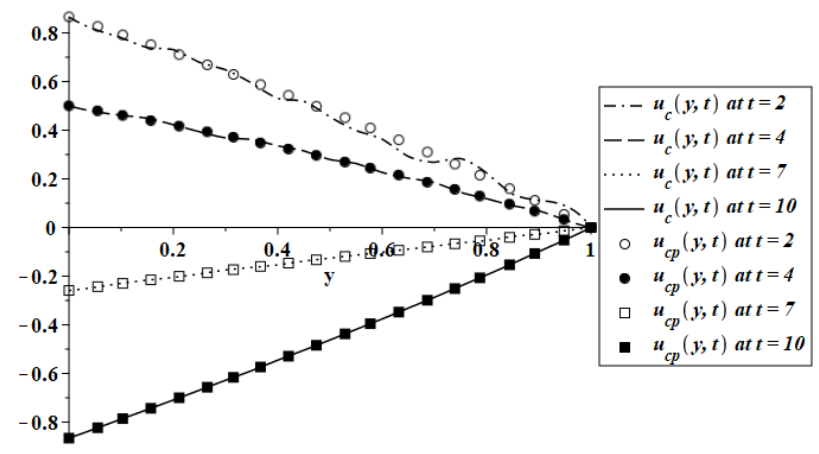

(A) $\mathrm{We}=0.3$

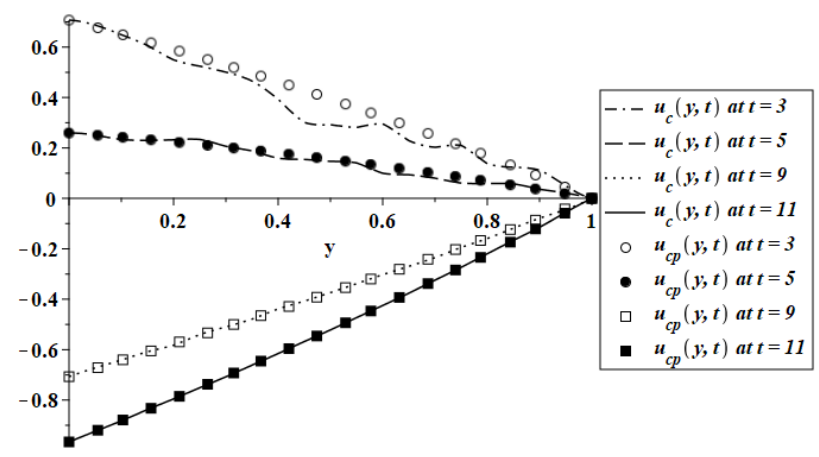

(B) $\mathrm{We}=0.7$

Figure (2) Convergence of starting solution $u_{c}(y, t)$ (numerical solution) to its permanent component $u_{c p}(y, t)$ given by Eq. (3.20) for $\alpha=0.95, \omega=\pi / 12$ and two values of We.

$\omega=\pi / 12$ and $\mathrm{We}=0.2$ and three different values of the pressure-viscosity coefficient $\alpha$. The oscillatory specific features of the two motions are better underlined and the oscillations' amplitude diminishes for decreasing values of the parameter $\alpha$. As it was to be expected, the order of magnitude of the oscillations' amplitude is the same for both motions and the phase difference is clearly observed.

\section{References}

[1] Bair, S., Jarzynski, J., Winer, W.Q., The temperature, pressure and time dependence of lubricant viscosity, Tribol. Int., 34(2001), 461-468.

[2] Barus, C., Isothermals, isopiestics and isometrics relative to viscosity, Am. J. Sci., 45(1893), 87-96.

[3] Bridgman, P.W., The Physics of High Pressure, MacMillan Company, New York, 1931.

[4] Cui, H.H., Silber-Li, Z., Zhu, S.N., Flow characteristics of liquids in microtubes driven by a high pressure, Phys. Fluids, 16(2004), 1803-1810. 

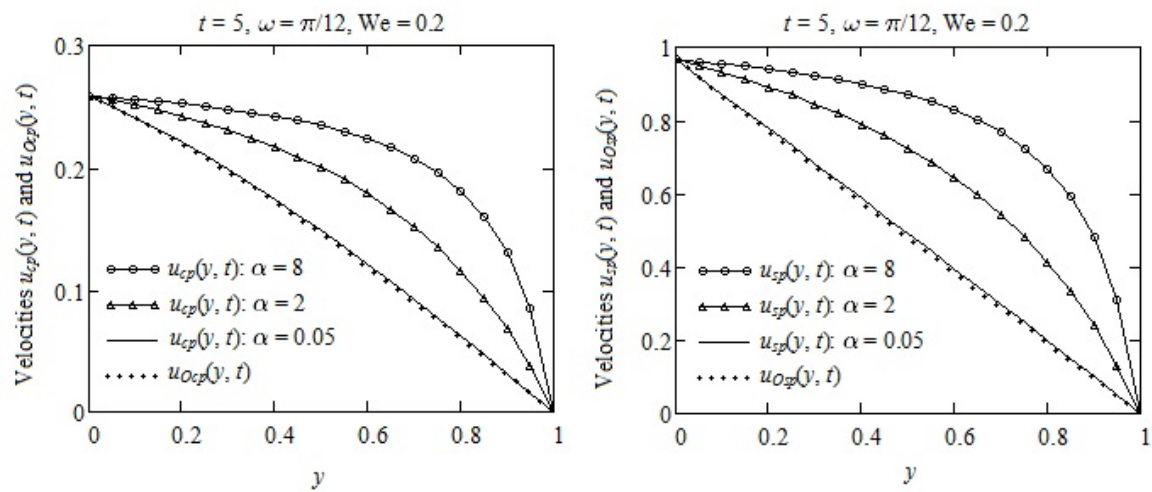

Figure (3) Convergence of the permanent solutions $u_{c p}(y, t)$ and $u_{s p}(y, t)$ given by Eqs. (3.12), respectively (3.13) to $u_{O c p}(y, t)$ and $u_{O s p}(y, t)$.
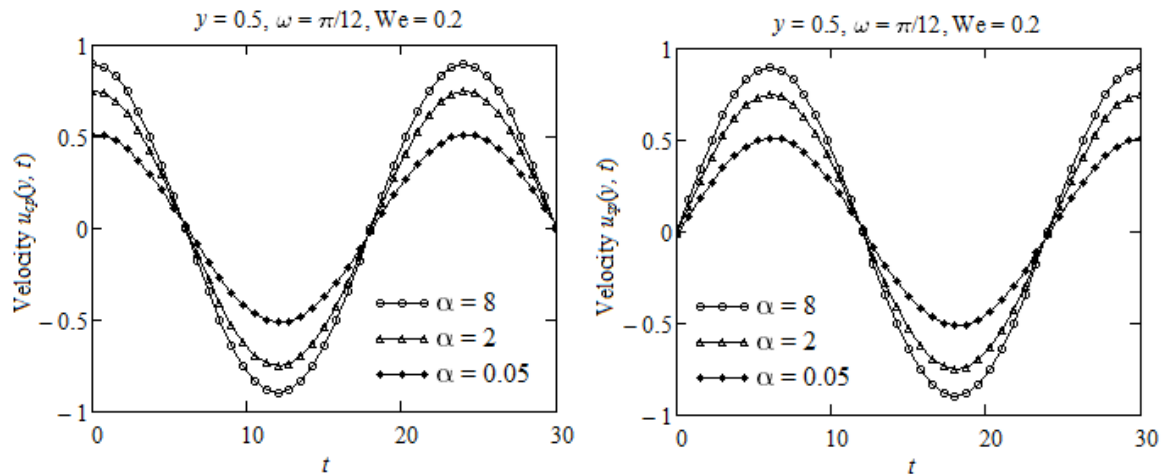

FiguRE (4) Time variation of the mid plane permanent velocities $u_{c p}(0.5, t)$ and $u_{s p}(0.5, t)$ given by Eqs. $(3.12)$, respectively (3.13) for three values of $\alpha$.

[5] Dealy, J.M., Wang, J., Melt Rheology and its Applications in the Plastic Industry, second ed., Springer, Dordrecht, 2013.

[6] Fetecau, C., Agop, M., Exact solutions for oscillating motions of some fluids with powerlaw dependence of viscosity on the pressure, Ann. Acad. Rom. Sci. Ser. Math. Appl., 12(2020), 295-311.

[7] Fetecau, C., Vieru, D., Exact solutions for unsteady motion between parallel plates of some fluids with power-law dependence of viscosity on the pressure, Applications in Engineering Science, 1(2020), 100003.

[8] Goubert, A., Vermout, J., Moldenaers, P., Gottfert, A., Ernst, B., Comparison of measurement techniques for evaluating the pressure dependence of the viscosity, Appl. Rheol., 11(2001), 26-37. 
[9] Griest, E.M. Webb, W., Schiessler, R.W., Effect of pressure on viscosity of high hydrocarbons and their mixture, J. Chem. Phys., 29(1950), 711-720.

[10] Housiadas, K.D., An exact analytical solution for viscoelastic fluids with pressuredependent viscosity, J. Non-Newton. Fluid Mech., 223(2015), 147-156.

[11] Housiadas, K.D., Internal viscoelastic flows for fluids with exponential type pressuredependent viscosity and relaxation time, J. Rheol., 59(2015), 769-791.

[12] Karra, S., Prusa, V., Rajagopal, K.R., On Maxwell fluids with relaxation time and viscosity depending on the pressure, Int. J. Non Linear Mech., 46(2011), 819-827.

[13] Malek, J., Rajagopal, K.R., Mathematical properties of the equations governing the flow of fluids with pressure and shear rate dependent viscosities, Handbook of Mathematical Fluid Dynamics, In: S. Friedlander, S. Serre (Eds.), Elsevier, Amsterdam, vol. 4, 2006, 407-444.

[14] Park, H.E., Lim, S.T., Laun, H.M., Dealy, J.M., Measurement of pressure coefficient of melt viscosity: drag flow versus capillary flow, Rheol. Acta, 47(2001), 1023-1038.

[15] Prusa, V., Revisiting Stokes first and second problems for fluids with pressure-dependent viscosities, Int. J. Eng. Sci., 48(2010), 2054-2065.

[16] Prusa, V., Srinivasan, S., Rajagopal, K.R., Role of pressure dependent viscosity in measurements with falling cylinder viscometer, Int. J. Non Linear Mech., 47(2012), 743-750.

[17] Rajagopal, K.R., Couette flows of fluids with pressure dependent viscosity, Int. J. Appl. Mech. Eng., 9(3)(2004), 573-585.

[18] Rajagopal, K.R., A semi-inverse problem of flows of fluids with pressure dependent viscosities, Inverse Probl. Sci. Eng., 16(2008), 269-280.

[19] Rajagopal, K.R., Saccomandi G., Vergori, L., Unsteady flows of fluids with pressure dependent viscosity, J. Math. Anal. Appl., 404(2013), 362-372.

[20] Stemmer, K., Hander, H., Hansen, U., A new method to stimulate convection with strong temperature and pressure-dependent viscosity in a spherical shell: applications to the Earth' mantle, Phys. Earth. Planet. Inter., 157(2006), 223-249.

[21] Stokes, G.G., On the theories of the internal friction of fluids in motion, and motion of elastic solids, Trans. Camb. Phil. Soc., 8(1845), 287-305.

[22] Szeri, A.Z., Fluid Film Lubrication, Cambridge University Press, Cambridge, 1998.

Constantin Fetecau

"Academy of Romanian Scientists", Section of Mathematics,

54 Splaiul Independentei,

RO-050094, Bucharest, Romania

e-mail: c_fetecau@yahoo.com

Abdul Rauf

"Air University Islamabad",

Department of Computer Science and Engineering,

Multan Campus (60000), Pakistan

e-mail: attari_ab092@yahoo.com 